\title{
$(4 E, 6 E, 12 E)$-Tetradecatriene-8,10-diyne-1,3-diyl Diacetate in Rat Plasma and Tissues by HPLC-UV Method and Their Application to a Pharmacokinetic and Tissue Distribution Study
}

\author{
Yan Huo, Yu-Qiang Liu, Zhong-Xu Bai, and Qian Cai \\ School of Medicine, Liaoning University of Traditional Chinese Medicine, Dalian 116600, China \\ Correspondence should be addressed to Qian Cai; caiqianmail@sina.com
}

Received 31 October 2013; Accepted 21 January 2014; Published 24 February 2014

Academic Editor: Yu-Ming Fan

Copyright (C) 2014 Yan Huo et al. This is an open access article distributed under the Creative Commons Attribution License, which permits unrestricted use, distribution, and reproduction in any medium, provided the original work is properly cited.

In China Atractylodis Rhizoma is widely used for the treatment of rheumatic diseases and digestive disorders. Stir-frying with wheat bran is the most common processing method. In order to clarify the influence of processing on pharmacological properties ofAtractylodis Rhizoma, an investigation was carried out to compare the pharmacokinetics and tissue distribution of typical constituent after oral administration of raw Atractylodis Rhizoma and processed ones. A simple, rapid, and sensitive high performance liquid chromatography with UV detection was developed and validated for the determination of $(4 E, 6 E, 12 E)$ tetradecatriene-8,10-diyne-1,3-diyl diacetate in rat plasma. A chromatography was carried out on Diamonsil $\mathrm{C}_{18}(250 \times 4.6 \mathrm{~mm}$; $5 \mu \mathrm{m})$ analytical column, using a mobile phase which consisted of acetonitrile and $0.1 \%$ phosphoric acid water $(60: 40$, v/v) at a flow rate of $1.0 \mathrm{~mL} \cdot \mathrm{min}^{-1}$. The wavelength was set at $336 \mathrm{~nm}$. The LLOQ of $(4 E, 6 E, 12 E)$-tetradecatriene-8,10-diyne-1,3-diyl diacetate was $0.00143 \mu \mathrm{g} \cdot \mathrm{mL}^{-1}$. Both accuracy and precision were satisfactory. The pharmacokinetic results showed that the $T_{\max }$ was 1 hour in advance and the $C_{\max }$ was increased after processing. Tissue distribution showed that the highest level was in spleen. And the concentrations in the spleen were increased after stir-frying with bran.

\section{Introduction}

In traditional Chinese medicine, Atractylodis Rhizoma is the dried root and stems from Atractylodes lancea (Thunb.) DC. or Atractylodes chinensis (DC.) Koidz. The medicinal herb is widely known as Cangzhu in China. And it is widely used for the treatment of rheumatic diseases, digestive disorders, mild diarrhea, and influenza [1]. In clinic, Atractylodis Rhizoma is often processed by stir-frying with wheat bran with the aim of reducing its dryness and increasing the function of tonifying spleen $[2,3]$.

Atractylodis Rhizoma is rich of essential oil including sesquiterpenes and polyethylene alkynes, which are the main active components in this medicine [4]. Recent researches have shown that polyethylene alkynes exhibit various desirable pharmacological effects including antiinflammatory, antibacterial, and antiarrhythmic activity [5]. $(4 E, 6 E, 12 E)$-tetradecatriene-8,10-diyne-1,3-diyl diacetate is one of polyethylene alkyne components. Some literature reported HPLC method for determination its content in Atractylodis Rhiomzoma [6-8].

However, there have been few methods available for its quantification in biosamples and few reports on its pharmacokinetic study and tissues distribution until now. The in vivo study of $(4 E, 6 E, 12 E)$-tetradecatriene-8,10-diyne1,3-diyl diacetate, an active component of Atractylodis Rhizoma, would be necessary and helpful for further clinical application and explanation of the processing mechanism. The present paper developed a new and simple RP-HP LC method for quantification of $(4 E, 6 E, 12 E)$-tetradecatriene8,10-diyne-1,3-diyl diacetate in rat plasma and tissues after oral administration of raw and processed Atractylodis Rhizoma, respectively. This fully validated method was successfully applied to a pharmacokinetic and tissue distribution study of $(4 E, 6 E, 12 E)$-tetradecatriene-8,10-diyne-1,3-diyl diacetate in rats for the first time. 


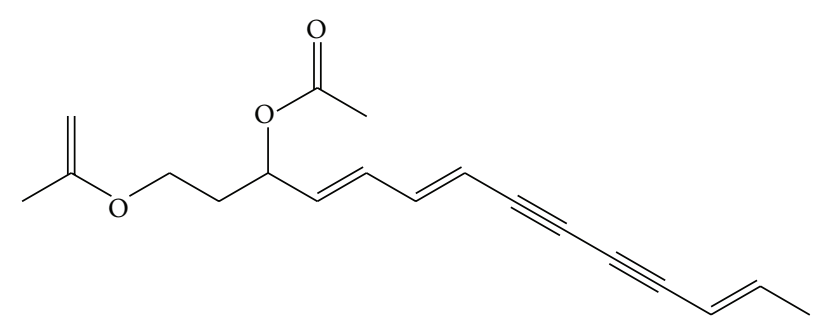

(a)

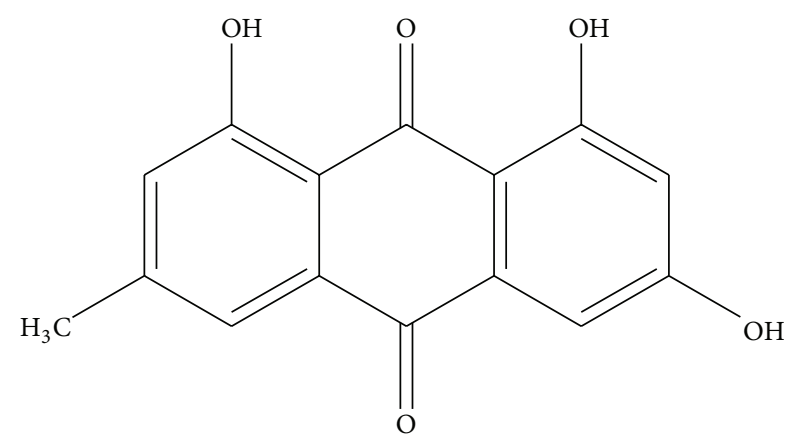

(b)

Figure 1: Chemical structures of $(4 E, 6 E, 12 E)$-tetradecatriene-8,10diyne-1,3-diyl diacetate (a) and Emodin (b).

\section{Experimental}

2.1. Chemicals and Reagents. The (4E,6E,12E)tetradecatriene-8,10-diyne-1,3-diyl diacetate (purity, 98\%) was supplied by Traditional Chinese Medicine Standardization Research Center (Shanghai, China). The IS called Emodin (purity, 98\%) was supplied by the National Institute for Food and Drug Control (Beijing, China). The chemical structures of $(4 E, 6 E, 12 E)$-tetradecatriene-8,10-diyne-1,3-diyl diacetate and IS are shown in Figure 1.

HPLC grade acetonitrile was purchased from Fisher Scientific Company (New Jersey, USA) and Pure water was supplied by Wahaha Company (Hangzhou, China). Analytical grade ethanol and chloroform were from Baierdi Company (Beijing, China). Atractylodis Rhizoma was identified by Professor Li Feng (Liaoning University of TCM) according to the standards of Chinese Pharmacopoeia 2010. The processed Atractylodis Rhizoma comes from the same batch Atractylodis Rhizoma. The herb was stored in a cool and dry place.

2.2. Preparation of Atractylodis Rhizoma Solution. Atractylodis Rhizoma (50 g) was crushed into powder and soaked into $600 \mathrm{~mL}$ of $80 \%$ ethanol for $24 \mathrm{~h}$ and then percolated at $2 \mathrm{~mL} \cdot \mathrm{min}^{-1}$, and Ethanol was evaporated to near dryness under reduced pressure to get the residue. Distilled water was added into the residue and then vortexed for $10 \mathrm{~min}$. The final concentration of Atractylodis Rhizoma solution was $2 \mathrm{~g} \cdot \mathrm{mL}^{-1}$ [9]. The sample was stored in dry and dark place before use.

2.3. Apparatus and HPLC Conditions. The liquid chromatographic system consisted of an LC-10 AD pump (Shimadzu, Kyoto, Japan) with a $20 \mu \mathrm{L}$ loop (Cotata, CA, USA) and an
SPD-10A ultraviolet-visile detector (Shimadzu, Kyoto, Japan). A LC-10 AD workstation was used for data acquisition. A Diamonsil $\mathrm{C}_{18}$ analytical column $(250 \times 4.6 \mathrm{~mm} ; 5 \mu \mathrm{m})$ from Dikma Technologies (Dalian, China) was used. The mobile phase consisted of acetonitrile and $0.1 \%$ phosphoric acid water $(60: 40, \mathrm{v} / \mathrm{v})$ at a flow rate of $1 \mathrm{~mL} \cdot \mathrm{min}^{-1}$. The detection wavelength was set at $336 \mathrm{~nm}$. All the measurements were performed at room temperature, and the injection volume was $20 \mu \mathrm{L}$.

2.4. Preparation of Standard Solution and Quality Control Samples. Stock solutions of $(4 E, 6 E, 12 E)$-tetradecatriene8,10-diyne-1,3-diyl diacetate and IS with concentrations of $0.143 \mathrm{mg} \cdot \mathrm{mL}^{-1}$ and $0.0504 \mathrm{mg} \cdot \mathrm{mL}^{-1}$, respectively, were prepared in methanol and stored at $-20^{\circ} \mathrm{C}$ and dark place until use. When we used the standard solution, the working concentration of $(4 E, 6 E, 12 E)$-tetradecatriene-8,10-diyne-1,3diyl diacetate and IS were $286 \mu \mathrm{g} \cdot \mathrm{L}^{-1}$ and $5.04 \mu \mathrm{g} \cdot \mathrm{L}^{-1}$, respectively. Calibration standards of $(4 E, 6 E, 12 E)$-tetradecatriene8,10-diyne-1,3-diyl diacetate were prepared by spiking the appropriate amount of the working solutions into $200 \mu \mathrm{L}$ blank rat plasma or tissue homogenates. To plasma samples, the final concentrations of calibration standard samples were $0.003575,0.00715,0.0143,0.03575,0.0715,0.143$, and $0.2145 \mu \mathrm{g} \cdot \mathrm{mL}^{-1}$. Quality control (QC) samples were prepared at low, medium, and high concentrations of 0.00375 , 0.03575 , and $0.0715 \mu \mathrm{g} \cdot \mathrm{mL}^{-1}$. To tissue homogenates, the final concentrations of calibration standard samples were $0.00143,0.003575,0.00715,0.0143,0.0286,0.03575$, and $0.0715 \mu \mathrm{g} \cdot \mathrm{mL}^{-1}$. Quality control (QC) samples were prepared at low, medium, and high concentrations of $0.00375,0.03575$, and $0.0715 \mu \mathrm{g} \cdot \mathrm{mL}^{-1}$ for tissue homogenates.

2.5. Sample Preparations. For plasma samples, the $200 \mu \mathrm{L}$ of rat plasma was mixed with $20 \mu \mathrm{L}$ IS $\left(0.00504 \mathrm{mg} \cdot \mathrm{mL}^{-1}\right)$. After protein was precipitated with $1,000 \mu \mathrm{L}$ of acetonitrile in $1.5 \mathrm{~mL}$ polypropylene tube by vortexing for $2 \mathrm{~min}$, the sample was centrifuged at $10,000 \mathrm{rpm} \cdot \mathrm{min}^{-1}$ for $5 \mathrm{~min}$. The supernatant was transferred into a $5.0 \mathrm{~mL}$ tube and added with $1,000 \mu \mathrm{L}$ of chloroform, extract and the under organic phase was transferred to another tube and evaporated to dryness at $40^{\circ} \mathrm{C}$ with nitrogen. The resulting extract was dissolved in $50 \mu \mathrm{L}$ of methanol, and vortex mixed for $2 \mathrm{~min}$. After centrifugation at $10,000 \mathrm{rpm} \cdot \mathrm{min}^{-1}$ for $5 \mathrm{~min}, 20 \mu \mathrm{L}$ supernatant was injected for analysis [10-12]. For tissue homogenate, each weighed tissue sample was thawed and the homogenized in ice-cold physiological saline $(2 \mathrm{~mL})$. Then a $200 \mu \mathrm{L}$ of tissue homogenate was taken and processed further like the plasma samples.

\subsection{Method Validation}

2.6.1. Specificity. The selectivity of the method was demonstrated by comparing chromatograms of blank plasma samples and tissue homogenate (without IS) obtained from rats and plasma samples and tissue homogenate spiked with the analytes and IS and plasma samples and tissue homogenate 


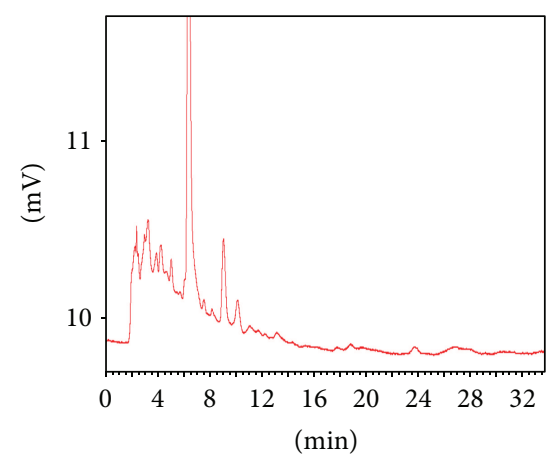

(a)

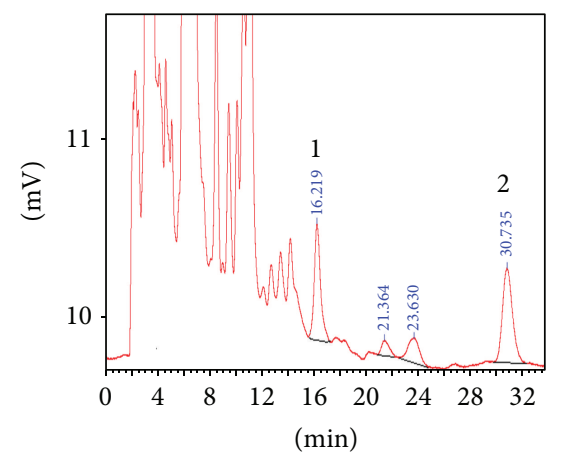

(d)

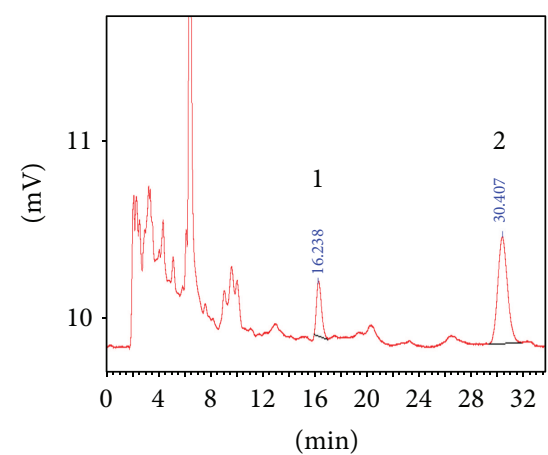

(b)

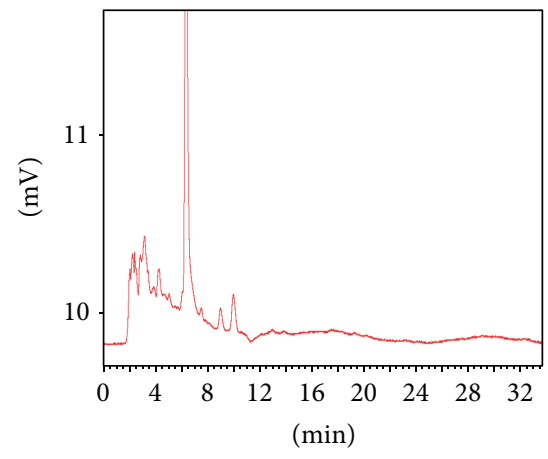

(e)

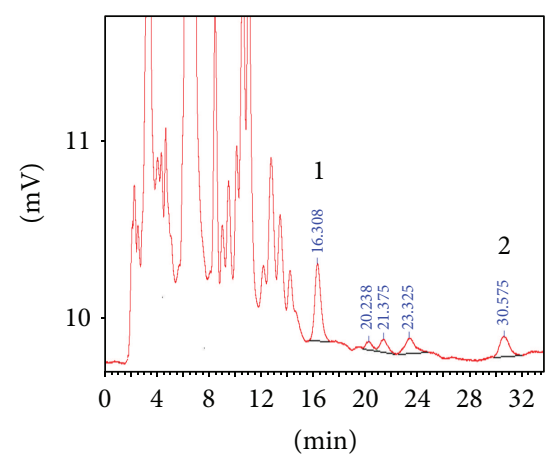

(c)

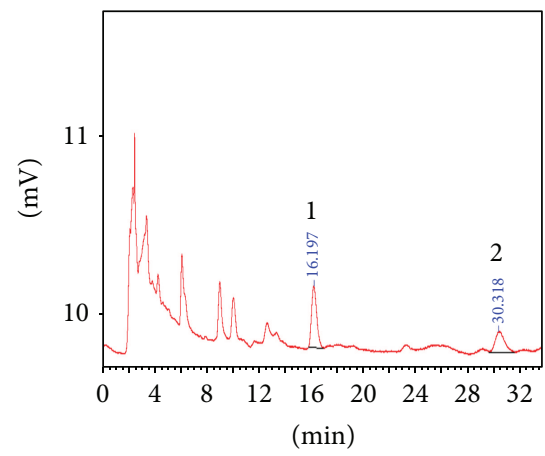

(f)

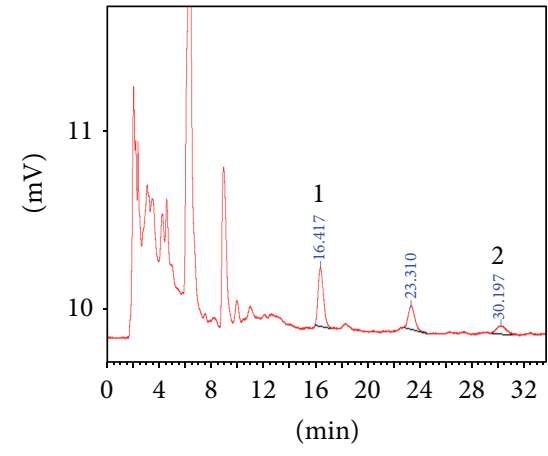

(g)

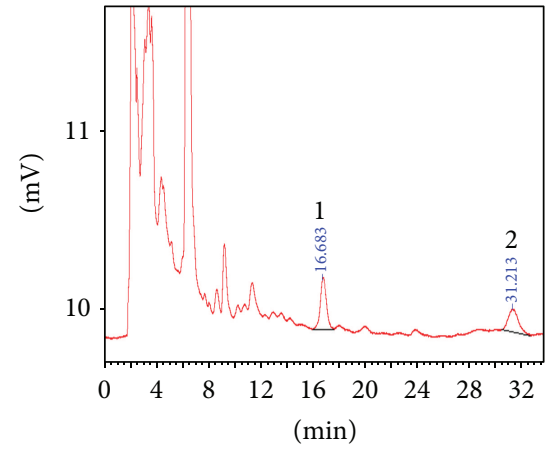

(h)

Figure 2: Chromatograms of blank plasma (a); blank plasma spiked with (4E,6E,12E)-tetradecatriene-8,10-diyne-1,3-diyl diacetate $20 \mu \mathrm{L}$ $\left(0.143 \mu \mathrm{g} \cdot \mathrm{mL}^{-1}\right)$ and IS $20 \mu \mathrm{L}\left(0.00504 \mathrm{mg} \cdot \mathrm{mL}^{-1}\right)(\mathrm{b})$; rat plasma sample $(4 \mathrm{~h})$ after oral administration of raw Atractylodis Rhizoma $40 \mathrm{~g} \cdot \mathrm{kg}^{-1}$

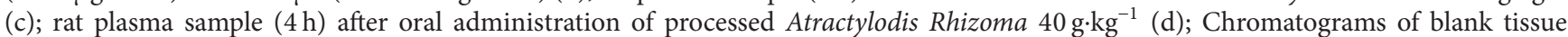
homogenate (e); blank tissue homogenate with (4E,6E,12E)-tetradecatriene-8,10-diyne-1,3-diyl diacetate $20 \mu \mathrm{L}\left(0.286 \mu \mathrm{g} \cdot \mathrm{mL}^{-1}\right)$ and IS $20 \mu \mathrm{L}$ $\left(0.00504 \mathrm{mg} \cdot \mathrm{mL}^{-1}\right)(\mathrm{f})$; spleen sample ( $\left.2 \mathrm{~h}\right)$ after oral administration of raw Atractylodis Rhizoma $40 \mathrm{~g} \cdot \mathrm{kg}^{-1}$ (g); spleen sample (2 h) after oral administration of processed Atractylodis Rhizoma $40 \mathrm{~g} \cdot \mathrm{kg}^{-1}$ (h).

after an oral dose. All blank plasma samples and tissue homogenates were prepared and analyzed to ensure the absence of interfering peaks.

2.6.2. Calibration Procedure. The linearity of the method was assessed by plotting calibration curves in plasma at seven concentration levels in triplicate on three consecutive days. The lower limit of quantification (LLOQ) was defined as the lowest concentration of the calibration curve that was measured with accuracy and precision by analyzing samples in six replicates at the concentration of $0.00143 \mu \mathrm{g} \cdot \mathrm{mL}^{-1}$ for $(4 E, 6 E, 12 E)$-tetradecatriene-8,10-diyne-1,3-diyl diacetate.

2.6.3. Accuracy and Precision. Intraday precision and accuracy were evaluated by analysis of the three QC samples with six determinations per concentration at the same day, whilst the interday precision and accuracy were measured over three consecutive days. The precision was defined as the relative standard deviation (RSD\%), while accuracy was determined by calculating the percentage deviation observed in the analysis of QC samples and expressed by relative 
TABLE 1: The Linear regression analysis of $(4 E, 6 E, 12 E)$-tetradecatriene-8,10-diyne-1,3-diyl diacetate in rat plasma and tissues $(n=7)$.

\begin{tabular}{lccc}
\hline Sample & Calibration curves & $r$ & Linear range \\
\hline Plasma & $Y=23.628 X+0.044$ & 0.9950 & $0.00361-0.18814$ \\
Heart & $Y=13.259 X+0.031$ & 0.9958 & $0.00149-0.07322$ \\
Liver & $Y=14.351 X+0.064$ & 0.9951 & $0.00144-0.06925$ \\
Spleen & $Y=12.936 X+0.028$ & 0.9951 & $0.00146-0.07431$ \\
Lungs & $Y=14.704 X+0.059$ & 0.9956 & $0.00143-0.07872$ \\
Kidney & $Y=12.936 X+0.028$ & 0.9951 & $0.00143-0.06716$ \\
Stomach & $Y=15.161 X+0.012$ & 0.9964 & $0.00143-0.06786$ \\
Large intestine & $Y=11.271 X+0.019$ & 0.9952 & $0.00146-0.08179$ \\
Small intestine & $Y=12.956 X+0.037$ & 0.9985 & $0.00144-0.07339$ \\
\hline
\end{tabular}

error $(\mathrm{RE} \%)$. The accepted criteria for the data were that the precision and accuracy should not exceed $15 \%$, except at the LLOQ, where it should not exceed $20 \%$.

2.6.4. Extraction Recovery and Stability. The extraction recoveries of $(4 E, 6 E, 12 E)$-tetradecatriene-8,10-diyne-1,3-diyl diacetate were determined at low, medium, and high level of QC samples. Recoveries were calculated by comparing the observed peak area ratios in biosamples to those nonprocessed standard solutions at the same concentrations. The recovery of IS was determined in the same way at the concentration of $0.00504 \mathrm{mg} \cdot \mathrm{mL}^{-1}$.

The stability of $(4 E, 6 E, 12 E)$-tetradecatriene-8,10-diyne1,3-diyl diacetate in plasma and tissue was determined under different storage or handling conditions. Short-term stability was assessed by analyzing QC samples kept at room temperature for $8 \mathrm{~h}$. Freeze-thaw stability was evaluated at three consecutive freeze-thaw cycles. Long-term stability was studied by assaying samples following a period of 10 days of storage at $-20^{\circ} \mathrm{C}$.

2.7. Applications in Pharmacokinetic Studies. All the studies on animals were in accordance with the Guidelines for the Care and Use of Laboratory Animals. Healthy SpragueDawley rats $(250 \pm 20 \mathrm{~g})$ were purchased from The Medical University of Dalian (Dalian, China) and acclimated in the laboratory for one week before to the experiments. Rats for oral ingestion were fasted for $12 \mathrm{~h}$ with free access to water. Rats were oral administration raw and processed Atractylodis Rhizoma at a single dose of $40 \mathrm{~g} \cdot \mathrm{kg}^{-1}$, respectively.

For plasma samples, twelve rats were randomly assigned to two groups for pharmacokinetic investigation $(n=6$ per group). The blood sample $(0.5 \mathrm{~mL})$ was collected at 0 , $0.17,0.33,0.5,1,2,3,4,6,8,12$, and $24 \mathrm{~h}$. All samples were immediately transferred into heparinized tubes and centrifuged for $5 \mathrm{~min}$ at $10,000 \mathrm{rpm} \cdot \mathrm{min}^{-1}$. The supernatant was stored at $-20^{\circ} \mathrm{C}$ and dark place until use.

2.8. Tissue Distribution Study. For tissue distribution study, forty-eight rats were divided into eight groups ( $n=6$ per group) randomly.

After oral administration four groups rats raw Atractylodis Rhizoma at a single dose of $40 \mathrm{~g} \cdot \mathrm{kg}^{-1}$ and the other four groups rats processed Atractylodis Rhizoma at a single dose of $40 \mathrm{~g} \cdot \mathrm{kg}^{-1}$. Heart, liver, spleen, lung, kidney, stomach, small intestine, and large intestine were collected at $0.5,2,4$, and $8 \mathrm{~h}$. Tissue samples were weighed $0.1 \mathrm{~g}$ rapidly, rinsed with physiological saline to remove the blood or content, blotted on filter paper, and then stored at $-20^{\circ} \mathrm{C}$ and dark place until use.

2.9. Statistical Analysis. HPLC analysis procedure was applied to analyze plasma concentration-time profiles of (4E,6E,12E)-tetradecatriene-8,10-diyne-1,3-diyl diacetate. Data was processed by noncompartmental method using Drug and Statistics (DAS) 2.0 software package (Chinese Pharmacological Society, Shanghai, China).

\section{Results and Discussion}

\subsection{Method Validation}

3.1.1. Specificity. The representative chromatograms for determination of $(4 E, 6 E, 12 E)$-tetradecatriene-8,10-diyne-1,3-diyl diacetate in plasma and tissues are shown in Figure 2. The retention time of Emodin (IS) was about $16.30 \mathrm{~min}$ and (4E,6E,12E)-tetradecatriene-8,10-diyne-1,3-diyl diacetate was about $30.45 \mathrm{~min}$. It was indicated that analytes and IS were well separated and no interferences were detected from endogenous substances or metabolites.

3.1.2. Linearity of Calibration Curve and Lower Limit of Quantification. The calibration curves were linear over the concentration range of $0.003575-0.2145 \mu \mathrm{g} \cdot \mathrm{mL}^{-1}$ in rat plasma and $0.00143-0.0715 \mu \mathrm{g} \cdot \mathrm{mL}^{-1}$ in tissue homogenates by weighted $\left(1 / x^{2}\right)$ linear least-squares regression method. The correlation coefficient values of the calibration curves were over 0.9900 . The RE of the back-calculated values of the standards from their nominal values were constantly within $15 \%$ for all values, including the LLOQ. The LLOQ measurement showed the respective averages $0.003575 \mu \mathrm{g} \cdot \mathrm{mL}^{-1}$ with $\mathrm{RSD} 7.68 \%$ for rat plasma and $0.00143 \mu \mathrm{g} \cdot \mathrm{mL}^{-1}$ with $\mathrm{RSD} 8.68 \%$ for tissue homogenates. Typical linear regression equations, correlation coefficients in plasma and each tissue were listed in Table 1.

3.1.3. Precision and Accuracy. The intraday and interday precision and accuracy were assessed by analyzing six aliquots of 
low, medium, and high concentration samples. The intraday precision of $(4 E, 6 E, 12 E)$-tetradecatriene-8,10-diyne-1,3-diyl diacetate in rat plasma and tissues ranged between 1.83 and $6.71 \%$ with RE of $-14.47 \sim-7.81 \%$ and the interday precision ranged between 1.57 and $8.68 \%$ with RE of $-14.38 \sim-5.13 \%$.

3.1.4. Recovery and Stability. The extraction recoveries of (4E,6E,12E)-tetradecatriene-8,10-diyne-1,3-diyl diacetate ranged from $86.6 \%$ to $91.6 \%$ in plasma and tissue samples and the method recovery ranged from $85.6 \%$ to $94.9 \%$, while the recovery of IS was above $80 \%$. These data indicated that the biosample preparation procedure was satisfied and can achieve the acceptable extraction recovery. Stability of analytes showed no significant sample loss over $12 \mathrm{~h}$ at room temperature, three freeze-thaw cycles, and 10 days storage condition. The RE of three conditions were within $\pm 15 \%$.

3.2. Pharmacokinetics of $(4 E, 6 E, 12 E)$-Tetradecatriene-8,10diyne-1,3-diyl Diacetate in Rats. The assay was applied to a preliminary pharmacokinetic experiment after oral administration of $40 \mathrm{~g} \cdot \mathrm{kg}^{-1}$ raw and processed Atractylodis Rhizoma to rats, respectively. Mean concentration-time curves were shown in Figures 3 and 4. The pharmacokinetic parameters were shown in Table 2.

A significant result of this study is finding that $(4 E, 6 E, 12 E)$-tetradecatriene-8,10-diyne-1,3-diyl diacetate showed double peaks after oral administration, which demonstrated that a hepatoenteral circulation may exist. For raw Atractylodis Rhizoma the absorption peaks in rat plasma was at $2 \mathrm{~h}$ and $4 \mathrm{~h}$, respectively and the $C_{\max }$ was $38 \pm 24 \mu \mathrm{g} \cdot \mathrm{L}^{-1}$. And for processed Atractylodis Rhizoma the absorption peaks was at $1 \mathrm{~h}$ and $3 \mathrm{~h}$, respectively and the $C_{\max }$ is $42 \pm 17 \mu \cdot \mathrm{gL}^{-1}$. So the time of absorption peak was 1 hour in advance and the concentration of rat plasma was increased after processing. The value of $T_{\max }$ and $T_{1 / 2}$ indicated that the $(4 E, 6 E, 12 E)$-tetradecatriene-8,10-diyne-1,3-diyl diacetate was rapidly distributed but slowly eliminated. The reason for this result also requires further study.

3.3. Tissue Distribution Study. The tissue concentrations of $(4 E, 6 E, 12 E)$-tetradecatriene-8,10-diyne-1,3-diyl diacetate determined at $0.5,2,4$, and $8 \mathrm{~h}$ after oral administration raw and processed Atractylodis Rhizomaat a dose of $40 \mathrm{~g} \cdot \mathrm{kg}^{-1}$ are shown in Table 3 and Figures 5 and 6 , which indicated that $(4 E, 6 E, 12 E)$-tetradecatriene-8,10-diyne1,3-diyl diacetate could be distributed to all collected tissues, for raw Atractylodis Rhizoma, the concentrations of $(4 E, 6 E, 12 E)$-tetradecatriene-8,10-diyne-1,3-diyl diacetate in tissue were distributed, followed by spleen, liver, small intestine, heart, stomach, large intestine, kidney, and lungs; for processed Atractylodis Rhizoma, the concentrations of $(4 E, 6 E, 12 E)$-tetradecatriene-8,10-diyne-1,3-diyl diacetate in tissue were distributed, followed by spleen, heart, kidney, liver, lungs, stomach, small intestine, large intestine. Relatively, the concentrations of $(4 E, 6 E, 12 E)$-tetradecatriene8,10-diyne-1,3-diyl diacetate of raw and processed Atractylodis Rhizoma was higher in the spleen. And the concentrations in the spleen were increased after stir-frying with

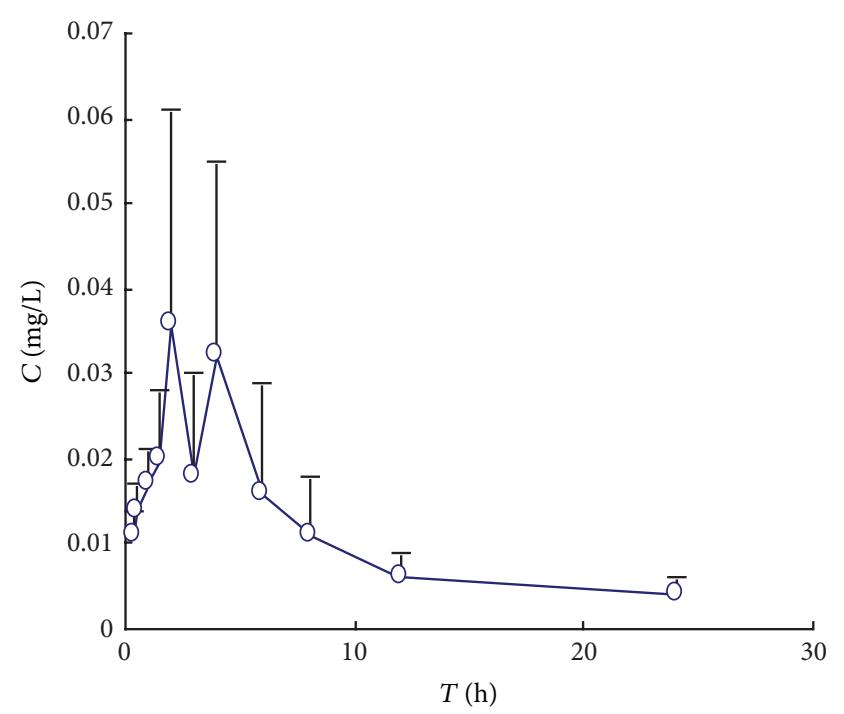

Figure 3: Mean plasma concentration-time curve of $(4 E, 6 E, 12 E)$ tetradecatriene-8,10-diyne-1,3-diyl diacetate after oral administration of raw Atractylodis Rhizoma $\left(40 \mathrm{~g} \cdot \mathrm{kg}^{-1}\right)$ to rats. (mean $\pm \mathrm{SD}$, $n=6)$.

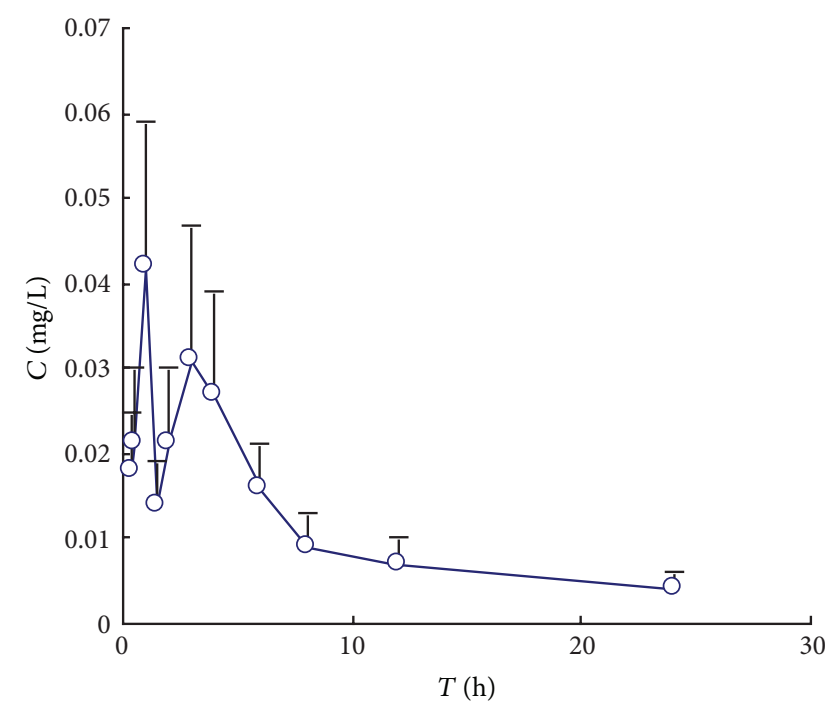

Figure 4: Mean plasma concentration-time curve of $(4 E, 6 E, 12 E)$ tetradecatriene-8,10-diyne-1,3-diyl diacetate after oral administration of processed Atractylodis Rhizoma $\left(40 \mathrm{~g} \cdot \mathrm{kg}^{-1}\right)$ to rats. (mean \pm $\mathrm{SD}, n=6)$.

bran. The results showed that processing can promote the rate of absorption of $(4 E, 6 E, 12 E)$-tetradecatriene-8,10-diyne-1,3diyl diacetate.

\section{Conclusion}

A simple, specific, and rapid RP-HPLC method with UV detection for quantification of $(4 E, 6 E, 12 E)$-tetradecatriene8,10-diyne-1,3-diyl diacetate in rat plasma has been developed for the first time. It has been successfully applied 
TABLE 2: The pharmacokinetic parameters of $(4 E, 6 E, 12 E)$-tetradecatriene-8,10-diyne-1,3-diyl diacetate of raw and processed AtractylodisRhi$z o m a$ at a dose of $40 \mathrm{~g} \cdot \mathrm{kg}^{-1}$ to rats, respectively $(n=6)$.

\begin{tabular}{lcr}
\hline Parameters & Raw & Processed \\
\hline $\mathrm{AUC}_{(0-t)}\left(\mathrm{mg} \cdot \mathrm{h} \cdot \mathrm{L}^{-1}\right)$ & $0.260 \pm 0.137$ & $0.265 \pm 0.100$ \\
$\mathrm{AUC}_{(0-\infty)}\left(\mathrm{mg} \cdot \mathrm{h} \cdot \mathrm{L}^{-1}\right)$ & $0.317 \pm 0.157$ & $0.303 \pm 0.111$ \\
$\mathrm{MRT}_{(0-t)}(\mathrm{h})$ & $7.706 \pm 0.888$ & $7.384 \pm 0.86$ \\
$\mathrm{MRT}_{(0-t)}(\mathrm{h})$ & $14.86 \pm 6.813$ & $12.22 \pm 3.364$ \\
$t_{1 / 2 z}(\mathrm{~h})$ & $9.905 \pm 7.382$ & $8.047 \pm 3.347$ \\
$T_{\max }(\mathrm{h})$ & $2.333 \pm 0.816$ & $1.000 \pm 0.817$ \\
$C_{\max }\left(\mathrm{mg} \cdot \mathrm{L}^{-1}\right)$ & $0.038 \pm 0.024$ & $0.042 \pm 0.017$ \\
\hline
\end{tabular}

TABLE 3: The tissue concentrations of $(4 E, 6 E, 12 E)$-tetradecatriene-8,10-diyne-1,3-diyl diacetate after oral administration raw and processed Atractylodis Rhizoma at a dose of $40 \mathrm{~g} \cdot \mathrm{kg}^{-1}$ to rats, respectively $(\mu \mathrm{g} / \mathrm{g})$.

\begin{tabular}{lcccccccc}
\hline \multirow{2}{*}{ Tissues } & \multicolumn{3}{c}{$0.5 \mathrm{~h}$} & \multicolumn{3}{c}{$2 \mathrm{~h}$} & \multicolumn{2}{c}{$4 \mathrm{~h}$} \\
& Raw & Processed & Raw & Processed & Raw & Processed & Raw & Processed \\
\hline Heart & 125.31 & 137.68 & 128.97 & 244.30 & 77.35 & 61.62 & 35.94 & 30.56 \\
Liver & 80.37 & 158.37 & 155.10 & 208.56 & 158.07 & 173.38 & 115.10 & 128.98 \\
Spleen & 170.71 & 119.41 & 253.18 & 292.69 & 186.45 & 204.92 & 17.34 & 30.66 \\
Lung & 69.35 & 70.01 & 46.54 & 174.26 & 84.79 & 100.26 & 40.20 & 60.61 \\
Kidney & 108.76 & 105.54 & 76.15 & 242.21 & 81.83 & 84.78 & 24.51 \\
Stomach & 112.31 & 107.95 & 109.77 & 122.96 & 65.22 & 78.53 & 43.17 & 59.93 \\
Large Intestine & 20.33 & 30.68 & 79.02 & 54.78 & 112.25 & 143.34 & 166.77 & 176.64 \\
Small Intestine & 91.25 & 106.29 & 139.04 & 115.53 & 191.22 & 159.02 & 44.91 & 54.78 \\
\hline
\end{tabular}

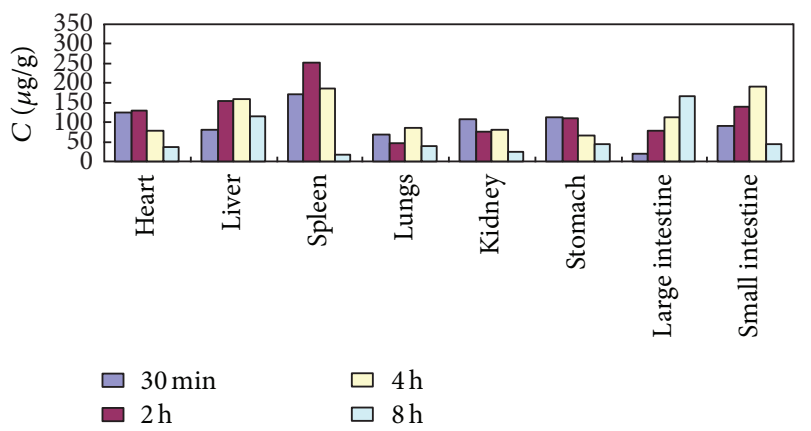

Figure 5: The concentration-time profile of $(4 E, 6 E, 12 E)$ tetradecatriene-8,10-diyne-1,3-diyl diacetate in tissues after oral administration of raw Atractylodis Rhizoma at a dose of $40 \mathrm{~g} \cdot \mathrm{kg}^{-1}$ to rats $(n=6)$.

to a preliminary pharmacokinetic and tissue distribution study of $(4 E, 6 E, 12 E)$-tetradedecatriene-8,10-diyne-1,3diyl diacetate after oral administration of $40 \mathrm{~g} \cdot \mathrm{kg}^{-1}$ raw and processed Atractylodis Rhizoma, respectively. We found that the $T_{\max }$ have significant difference $(P<0.05)$, and other pharmacokinetics have no significant difference after using Student's $t$-test. The result indicated that processing can promote and accelerate the absorption and the concentration of $(4 E, 6 E, 12 E)$-tetradecatrinen-8,10-diyne-1,3-diyl diacetate is the highest in the spleen Which proved that the traditional theory of processing Atractylodis Rhizoma can increase its function of tonifying the spleen.

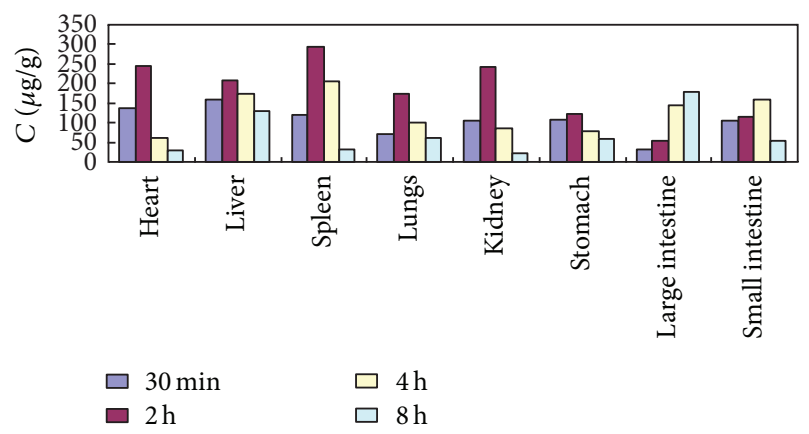

Figure 6: The concentration-time profile of $(4 E, 6 E, 12 E)$ tetradecatriene-8,10-diyne-1,3-diyl diacetate in tissues after oral administration of processed Atractylodis Rhizoma at a dose of $40 \mathrm{~g} \cdot \mathrm{kg}^{-1}$ to rats $(n=6)$.

\section{Conflict of Interests}

The authors declare that they have no conflict of interests.

\section{Acknowledgment}

This project was supported by grants from the National Natural Science Foundation of China (no. 81202919) and the Special Scientific Research for Traditional Chinese Medicine of State Administration of Traditional Chinese Medicine of China (no. 20110700712). 


\section{References}

[1] Chinese Pharmacopoeia Committee, Pharmacopeia of People's Republic of China, Chinese Medicine Science and Technology, Beijing, China, 2010.

[2] S. S. Pu, Z. X. Pu, and S. T. Yuan, "Study on processing historical evolution of Atractylodes Lancea," Chinese Pharmaceutical Journal, vol. 35, no. 11, pp. 87-91, 2000.

[3] Y. J. Liu, L. Y. Xu, and S. T. Yuan, "Study on processing technology of stir-frying atractylodes with wheat bran," Chinese Journal of Hospital Pharmacy, vol. 29, no. 15, pp. 1267-1268, 2009.

[4] M. Resch, A. Steigel, Z.-L. Chen, and R. Bauer, "5-lipoxygenase and cyclooxygenase-1 inhibitory active compounds from Atractylodes lancea," Journal of Natural Products, vol. 61, no. 3, pp. 347-350, 1998.

[5] M. Resch, J. Heilmann, A. Steigel, and R. Bauer, "Further phenols and polyacetylenes from the rhizomes of Atractylodes lancea and their anti-inflammatory activity," Planta Medica, vol. 67, no. 5, pp. 437-442, 2001.

[6] C. W. Fang, M. Fan, S. J. Liu, and L. B. Pan, "Simultaneous determination of three acetylenic polyethylene components in atractylodes lancea," Journal of Chinese Medicinal Materials, vol. 33, no. 6, pp. 932-934, 2010.

[7] Y. Q. Liu, T. Z. Jia, and Q. Cai, "Determination of three components in stir-frying Atractylodes Rhizoma with and without bran by HPLC," Chinese Traditional Patent Medicine, vol. 35, no. 1, pp. 131-135, 2013.

[8] Y. M. Chen, G. X. Chou, and Z. T. Wang, "Simultaneous determination of polyacetylene components in Cangzhu by reversed-phase high performance liquid chromatography," Chinese Journal of Chromatography, vol. 25, no. 1, pp. 84-87, 2007.

[9] Y. S. Zhang, Z. M. Wang, J. J. Zhu, B. Chen, and Y. Q. Li, "Determination of atractylodin in rat plasma by HPLC-UV method and its application to a Pharmacokinetic Study," Journal of Liquid Chromatography and Related Technologies, vol. 35, no. 6, pp. 778-787, 2012.

[10] D. F. Liu, L. Zhang, and T. Chen, "Pharmacokinetics Study on Paeoniflorin in Silli San," China Jornal of Experimental Traditional Medical Formulae, vol. 11, no. 2, pp. 36-38, 2005.

[11] S.-Z. Chen, H. Jia, Y.-H. Wu, and H. Wang, "Pharmacokinetics of honokiol in rats," Journal of Peking University, vol. 36, no. 1, pp. 41-44, 2004.

[12] J. M. Li, S. X. Huo, P. P. Gao Wu, X. M. Pang, Q. Lin, and M. Yan, "Pharmacokinetics study of galangin in rats," Chinese Pharmacological Bulletin, vol. 28, no. 9, pp. 1307-1310, 2012. 

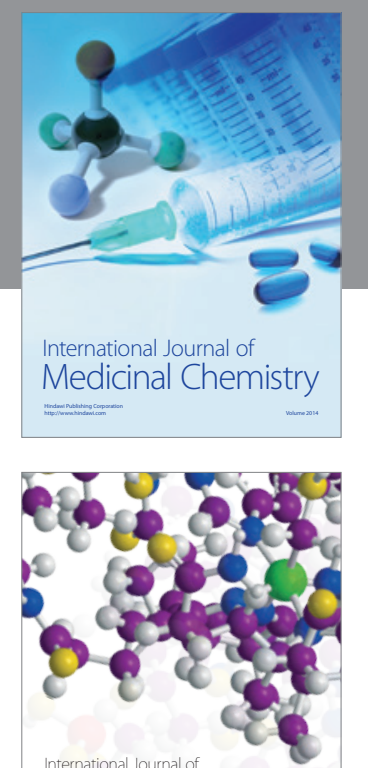

\section{Carbohydrate} Chemistry

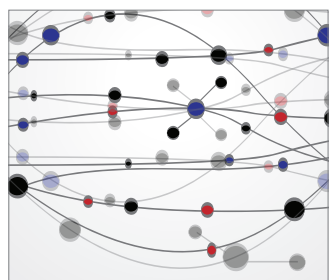

The Scientific World Journal
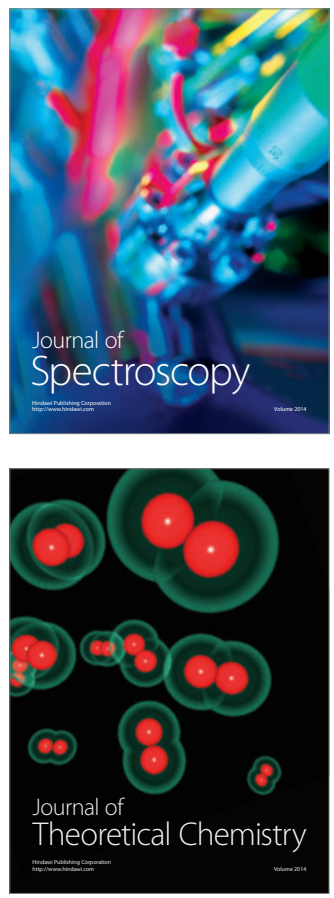
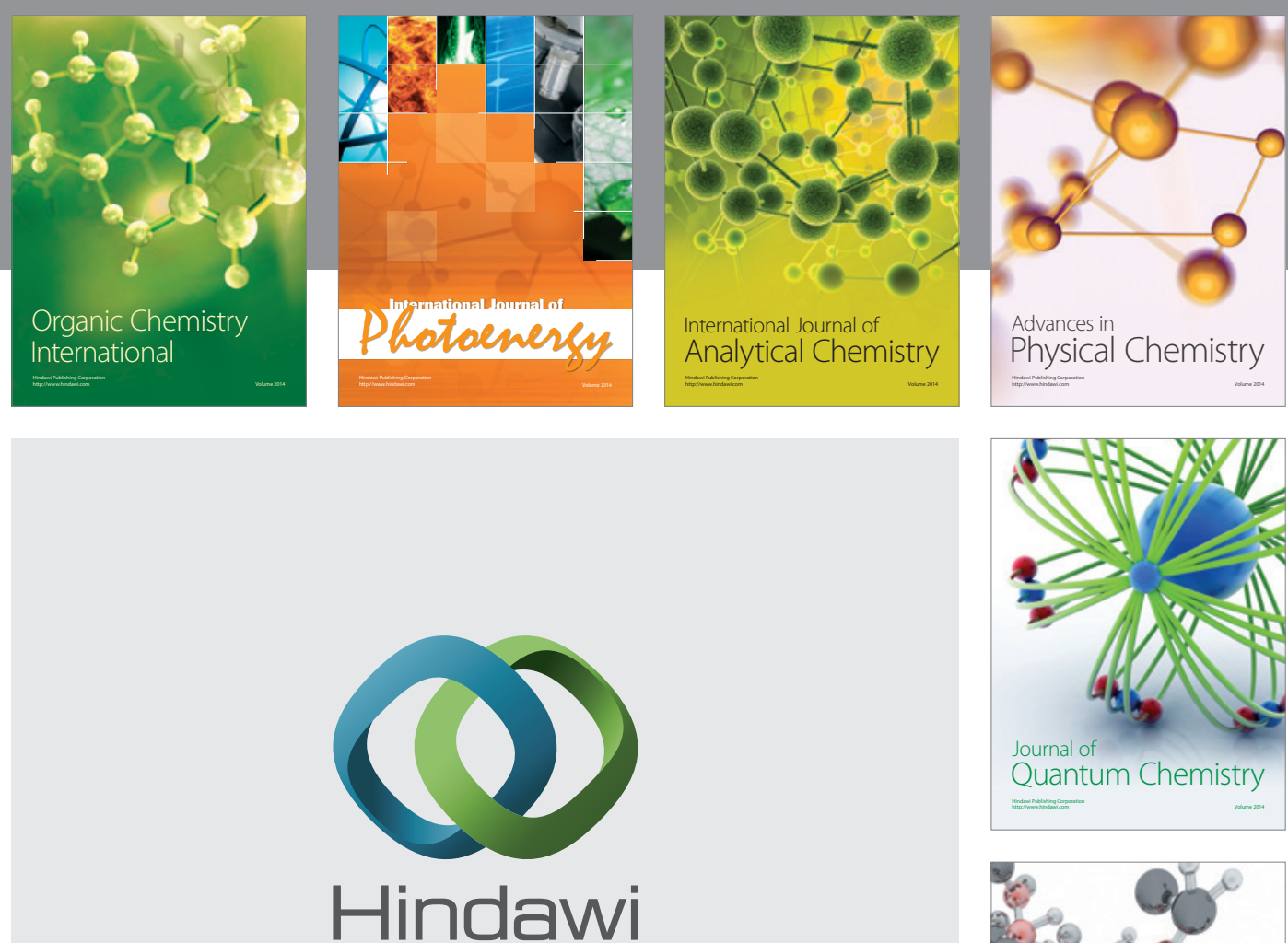

Submit your manuscripts at

http://www.hindawi.com

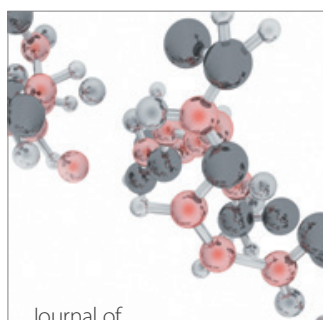

Analytical Methods

in Chemistry

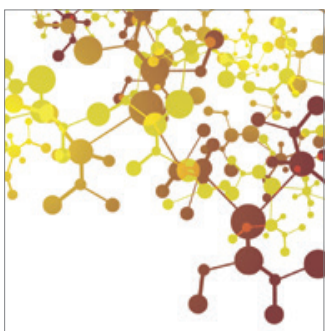

Journal of

Applied Chemistry

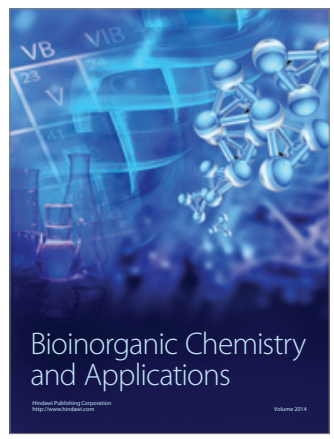

Inorganic Chemistry
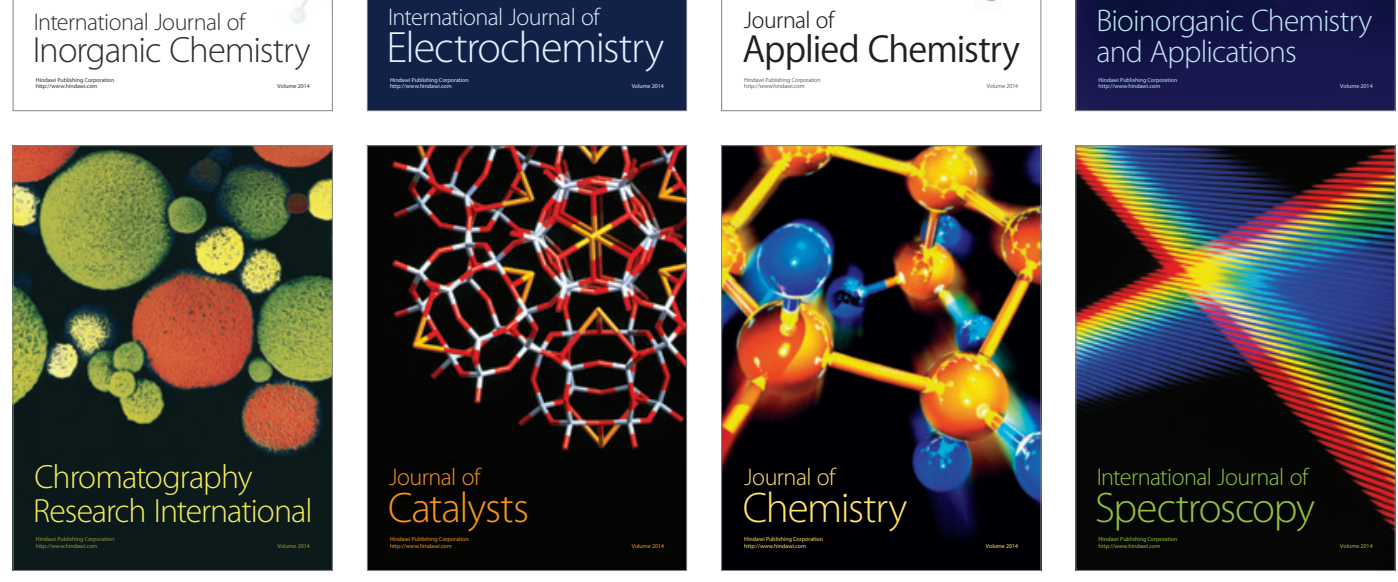